\title{
Constitutions des Etats de la Caraibe
}

Costa Rica

\section{Claude Emeri}

\section{(2) OpenEdition Journals}

Édition électronique

URL : http://journals.openedition.org/plc/842

DOI : $10.4000 /$ plc. 842

ISSN : 2117-5209

Éditeur

L'Harmattan

\section{Édition imprimée}

Date de publication : 1 janvier 2010

Pagination : 201-208

ISBN : 978-2-296-1141-3

ISSN : 1279-8657

Référence électronique

Claude Emeri, «Constitutions des Etats de la Caraibe », Pouvoirs dans la Caraïbe [En ligne], 16 | 2010, mis en ligne le 06 mai 2011, consulté le 20 avril 2019. URL : http://journals.openedition.org/plc/842 DOI : $10.4000 /$ plc. 842

Ce document a été généré automatiquement le 20 avril 2019.

(c) Pouvoirs dans la Caraïbe 


\title{
Constitutions des Etats de la Caraibe
}

\author{
Costa Rica
}

\section{Claude Emeri}

1 Statocratie et présidentialisme sont les deux clés d'interprétation du mimétisme constitutionnel latino-américain. Sous la réserve majeure, que nous rappellent Pierre Bon et Franck Moderne (Blanquer 2001) : la règle du jeu est fondamentalement tributaire de son environnement. C'est dans ces conditions que l'on peut épouser l'aphorisme selon lequel Costa Rica incarne "l'exception centraméricaine », le seul Etat de démocratie constitutionnelle-pluraliste de l'isthme, cerné par des dictatures populistes ou prétoriennes intermittentes. «La Suisse de l'Amérique centrale », comme l'Uruguay était «la Suisse de l'Amérique latine » dans le discours récurrent des juristes libéraux. Il est pourtant vérifié que les garanties institutionnelles de la polyarchie, telles qu'énumérées par Robert Dahl, s'inscrivent au Costa Rica dans la durée : plus de cinquante ans sans crise politique majeure, scandés par des élections régulières, pluralistes, significatives; et par l'alternance au pouvoir de deux partis modérés dans leur orientation convergente vers le centre, des « partis du système » qui ne contestent pas les règles fondamentales du jeu.

2 Lijphart (1994) intègre alors Costa Rica dans le catalogue des vingt-sept Etats de la jurisphère qui méritent le qualificatif de démocratiques, seul sur le continent américain avec le Canada et les U.S.A.

3 Comment expliquer cette exception, ce miracle? Sans doute pas en se penchant sur les règles de droit politique traditionnelles; elles étaient depuis les origines d'inspiration étasunienne et n'ont pas freiné une instabilité chronique du système institutionnel jusqu'en 1948.

4 Avant cette date, rien ne différenciait Costa Rica de ses voisins; elle était, elle aussi, labellisée «République bananière », soumise à l'imperium gringo de la "Yunai » (United Fruit Company) et du Département d'Etat (Gamboa Guzman 1973). Mieux vaut alors se tourner vers les indicateurs d'ordre socio-culturel: ils enseignent d'abord que si le capitalisme agraire s'y est accompagné, comme ailleurs, d'une forte lutte des classes, celle-ci n'y a pas été démultipliée par la lutte des races; assez remarquable, l'homogénéité ethnique d'une population à plus de $85 \%$ de race blanche -ladinos solidement et séculairement implantée sur les hauts plateaux de la cordillère pacifique et 
dans le Valle Central qui abrite, entre 800 et 1400 mètres d'altitude, les riches zonas cafetaleras autour de San José, Cartago, Tres Rios et Alajuela; il n'y a quasiment plus d'Indiens et quelques milliers de noirs et de mulâtres venus des Antilles britanniques sont concentrés dans les plaines bananières de la côte Caraïbe (Lasserre 1977, 209). Cette population de petits propriétaires ruraux a entretenu, dès l'époque coloniale, des valeurs nationalistes et libérales qui se traduisent par deux traits complémentaires : un taux de scolarisation très élevé - l'Université de Costa Rica est dotée d'un statut constitutionnel (articles 84 et 85) - et un anticléricalisme très vif - le catholicisme est la religion de l'Etat (article 76) mais il est interdit d'utiliser les édifices religieux à des fins de propagande politique (article 28).

5 On y ajoute, spontanément, l'antimilitarisme; il y a eu des gouvernements militaires, mais plutôt de dictablanda et les ticos notaient fièrement « qu'il y a au Costa Rica plus d'instituteurs que de soldats »'. Depuis 1949, « l'armée est interdite en tant qu'institution permanente » (article 12), les acteurs politiques veillent avec un soin jaloux à ce que les moyens techniques des forces de police soient maintenus au strict niveau nécessaire à «la surveillance et la préservation de l'ordre public » (ibid.) et leurs autorités provinciales sont inéligibles à l'Assemblée législative (article 109-6).

6 Au-delà des symboles, mais sans négliger leur portée, l'analyse politique les réintègre dans une trilogie explicative séduisante (Dabène, 1992, 368): une lutte des classes neutralisée, un système politique consensualiste, ouvert au compromis et la division des pouvoirs. Nous mettrons bien sûr ici l'accent sur ce troisième axe d'approche, d'ordre institutionnel.

7 «Small is beautiful ». Costa Rica est un exemple pionnier de la vogue très contemporaine de l'Etat miniature: Charbonnier est maître chez soi. Isolés dans leurs « frais paysages de pinèdes et de plantations de café » (Gamboa G. 1973, 239), les josefinos, pauvres mais libres, n'avaient rien à attendre du Roi d'Espagne ou de ses officiers. Non plus que des Provinces, unies ou non, d'Amérique centrale ; ils épousèrent très vite et avec ferveur les valeurs libérales du constitutionnalisme; la trace s'en retrouve dans les principes fondamentaux du droit politique exprimés dès la Déclaration d'Indépendance de 1821 : celle-ci vient de Guatemala, les Costaricains l'ignorent quelque temps, repliés sur euxmêmes et hésitent entre deux offres fédéralistes, celle des Provinces Unies et celle d'Iturbide à Mexico.

8 En fin de compte, c'est le nationalisme qui l'emporte en 1848 avec l'enracinement d'une République indépendante, populiste mais profondément libérale.

9 Le socle du droit politique est incontestablement d'inspiration nord-américaine, agrémenté de nettes retouches à caractère nationaliste. On y repère les droits de l'homme dans leur formulation virginienne universaliste et individualiste (Titre IV) ; s'y ajoute une précaution légaliste pas inutile dans un contexte politique parfois tendu: le droit est énoncé ; puis les formes de sa garantie et, surtout, les conditions dans lesquelles la loi - et seule la loi - pourra en limiter l'étendue sont soigneusement précisées (cf. par exemple, l'article 24 dans sa dernière rédaction, méticuleuse, du 29 mai 1996). Ce culte de la norme trouve son expression la plus forte à l'article 10 de la Constitution : dans sa rédaction originelle, il posait solennellement la généralité du principe de constitutionnalité : « Les dispositions du pouvoir législatif ou du pouvoir exécutif contraires à la Constitution seront absolument nulles tout comme les actes de ceux qui usurpent les fonctions publiques et les nominations faites en dehors des formalités légales »; le contrôle de la constitutionnalité des lois et des décrets était confié à la Cour suprême statuant à la 
majorité des deux-tiers. Dans sa formulation actuelle - du 18 août 1989 -, l'article 10 confie ce soin à une Chambre spéciale de la Cour, statuant à la majorité absolue de ses membres, précisant en outre ses attributions en matière de conflit de compétences ou de révision constitutionnelle. A Costa Rica, l'Etat de droit est assurément l'Etat du droit.

Ce droit trouve une coloration sociale très forte dans le Titre $\mathrm{V}$ de la Constitution qui ne se contente pas d'énumérer les droits de la famille ou des travailleurs mais qui va jusqu'à préciser la durée maximale du temps de travail (article 58), la durée minimale des congés annuels (article 59) ... et le principe de la préférence nationale dans le contrat de travail (article 68). La référence au "principe chrétien de justice sociale » est explicite (article 74) et date de 1949.

11 On revient au modèle de Philadelphie dans une interprétation, rigide et présidentialiste du principe de séparation des pouvoirs. Chacun d'entre eux est clairement défini dans sa composition, organiquement indépendant des autres - ainsi : les membres de la famille du Président sont inéligibles à l'Assemblée (article 109-8 de la Constitution) et les magistrats de la Cour suprême sont inéligibles à la Présidence de la République (article 132-5) - ou soumis à des conditions de majorité qualifiée applicable à la révision constitutionnelle - ainsi, pour toucher à la composition de la Cour suprême (articles 157, 158) ou pour briser le veto présidentiel (article 127).

Dans l'exercice de leurs attributions respectives, on mettra l'accent sur l'indépendance renforcée du pouvoir judiciaire : il est soumis à la Constitution et à la loi (article 154), la responsabilité (disciplinaire) des juges ne peut être mise en cause que devant leurs pairs (articles 165 et 167). Au contraire, on constate que les pouvoirs gouvernemental et législatif ne sont pas totalement isolés l'un de l'autre: on a noté le droit de veto du Président sur les lois; dans l'autre sens, on doit citer, classique, le monopole de l'Assemblée en matière budgétaire - le veto ne peut être apposé au budget ordinaire (article 125). Surtout, plus original, le «fusible» que réalise la division du pouvoir exécutif entre le Président - et les vice-présidents, au nom de deux - d'une part, et les ministres du Gouvernement (articles 141 à 146); ces derniers sont nommés et révoqués par la Présidence, ont en charge un ou plusieurs départements ministériels et contresignent «les décrets, accords, décisions et ordres du pouvoir exécutif»; pour certains de ces actes (article 147), une délibération du « Conseil de Gouvernement » est requise. Comme, en parallèle, les ministres peuvent participer « avec voix consultative, aux sessions de l'Assemblée » (article 145) et qu'ils sont soumis, de même que le Président, à une procédure d'empêchement initiée par l'Assemblée (article 121-9), on imagine aisément un système d'accountability politique proche du modèle orléaniste ; un Président adroit, comme le fut José Figueres, peut alors jouer sur l'intérim (article 135) et le contreseing pour alimenter et apaiser la négociation avec l'Assemblée (Kantor, in DiBacco, 1977).

13 La citoyenneté est le troisième pilier sur lequel repose le système républicain costaricain; elle est minutieusement organisée afin d'interdire sa capture par une classe ou un clan. D'autant que le peuple souverain ne s'exprime que par la voie du mandat représentatif ; le referendum est ignoré - sauf, localement, pour modifier la carte des provinces (article 168) - sans doute pour éviter les dérives populistes, encore que l'élection présidentielle puisse être porteuse de germes plébiscitaires. Pour les éliminer, on notera la règle de la non-rééligibilité, définitive pour la Présidence (article 132) et de non-rééligibilité immédiate des députés (article 107) ; elles s'insèrent dans un dispositif qui vise à conjurer le népotisme et le caciquisme. 

suffrage au sens que lui donnait Hauriou : elle «fait sortir le corps électoral des brumes de la souveraineté $»^{2}$ et repose sur deux piliers solidaires : une conception intégratrice et une garantie juridictionnelle de la citoyenneté (Titre VIII). échappe puisque l'inscription sur la liste électorale s'effectue dès la naissance par le biais du Registre civil, sanctionnée par la possession obligatoire d'une carte d'identité - cédula de identitad - renouvelée automatiquement tous les dix ans, infalsifiable, indispensable dans la vie quotidienne et qui tient lieu de carte d'électeur (article 95-2 et 95-5). Et le vote est obligatoire - sans véritable sanction, il est vrai (article 93) - aboutissant à un taux de participation élevé, régulièrement croissant et aujourd'hui supérieur à $80 \%$ des inscrits. Mais le modèle costaricain réside surtout dans une institution très originale qui inspire aujourd'hui la plupart des Etats voisins : le Tribunal suprême des Elections (Titre VIII, chapitre 3, articles 99 à 104) ${ }^{3}$; il maîtrise l'intégralité du processus électoral «l'organisation, la direction et la surveillance des actes relatifs au suffrage »- depuis la confection des listes électorales jusqu'à la proclamation des résultats des scrutins nationaux, par l'émission d'actes de nature réglementaire ou juridictionnelle insusceptibles de recours (article 103). Trois à cinq magistrats titulaires et trois suppléants élus à la majorité des deux-tiers par la Cour suprême pour un mandat de six ans renouvelable et bénéficiant des privilèges et immunités des membres de cette Cour (article 101).

16 En pratique, ce Tribunal s'est avéré un instrument remarquable de moralisation des mœurs électorales et de garantie effective du pluralisme; il étend son emprise sur la législation électorale à propos de laquelle sa consultation est obligatoire et son avis assimilable à un veto (article 97) et sur les partis politiques qui doivent lui soumettre leurs comptes pour bénéficier du financement public de l'article 96 de la Constitution. Son autorité est incontestée de l'ensemble des entrepreneurs politiques.

17 Autant dire que si, à Costa Rica, la politique n'est pas « saisie par le droit », ce dernier est adossé sur le modèle polyarchique: les compétitions électorales s'y déroulent conformément à ses axiomes, à intervalles réguliers, dans des conditions régulières, comme la «lutte retenue » chère à Raymond Aron; elles sont couplées : le Président et l'Assemblée sont élus le même jour pour un identique mandat de quatre ans - non renouvelable, répétons-le, ce qui tempère la professionnalisation de la politique - selon deux modes de scrutin antagonistes; majoritaire à deux tours éventuels - un seul tour si un candidat obtient $40 \%$ des suffrages - pour l'élection présidentielle (article 138) ; proportionnel, afin de ménager la représentation des minorités et le pluralisme politique pour l'Assemblée (article 95-6 et 95-7).

18

On a imputé à cette simultanéité la « majoritarisation » du système de partis au bénéfice de deux d'entre eux polarisés autour du Président et de son principal concurrent: un bipartisme presque parfait, à l'étasunienne, cimenté par l'absence de partis hors système ; quelque temps interdit sur la base de l'article 98 de la Constitution, le Parti communiste n'a pas survécu au plan électoral à l'effondrement du modèle soviétique et l'influence du castrisme est d'ordre onirique. L'alternance interpelle deux formations centristes, de rassemblement, Partido Liberación Nacional et Partido Unitad Social Cristiana, dans «un spectre idéologique appauvri dans le sens d'une harmonie conservatrice $»^{4}$. 
Elle s'exprime à travers le pragmatisme et le réalisme de nombre de dispositions constitutionnelles relatives à l'action sociale de l'Etat et au contrôle de ses interventions ; celles qui ont trait au Trésor public et à l'Inspection générale de la République qui le surveille, ou aux institutions autonomes marquent bien le vécu polyarchique d'une société qui s'insère véritablement dans l'Etat de droit pratiqué au quotidien et pas seulement dans le discours des robins.

\section{BIBLIOGRAPHIE}

AMERINGER, Charles. Democracy in Costa Rica. New York : Praeger, 1982.

BLANQUER, Jean-Michel. "L’Amérique latine", Pouvoirs, n98, 2001, spécialement : BON, Pierre. "L'Etat en Amérique latine", p. 17 et s. et MODERNE, Franck. "Les avatars du présidentialisme dans les Etats latino-américains", p. 63 et s.

DABENE, Olivier. Costa Rica : Juicio a la democracia. San José : Flacso, 1992.

DABENE, Olivier. Amérique latine, la démocratie dégradée. Paris : éd. Complexe, 1997.

DIBACCO, Thomas V. Presidential Power in Latin American Politics. New York : Praeger, 1977.

GAMBOA GUZMAN, Francisco. Costa Rica. De la flibuste au Pentagone. Paris : Editions sociales, 1973.

JIMENEZ, Mario A. Desarollo constitucional de Costa Rica. San José : Juricentro, 1979.

LASSERRE, Guy. Les Amériques du Centre. $2^{\mathrm{e}}$ éd. Paris : PUF (coll. Magellan, 29), 1977.

LIJPHART, Arend. Electoral Systems and Party Systems : a study of Twenty-seven Democracies 1949-1990. Oxford : Oxford university Press, 1994.

\section{NOTES}

1. Mais, relevait dit-on le penseur et éducateur Omar Dengo, « C'est pour dissimuler une amère vérité : nous avons en réalité plus d'analphabètes que de balles de fusil! ». Pour autant, les dépenses publiques d'enseignement, dont le seuil minimal est constitutionnellement fixé à $6 \%$ du P.I.B. (article 78), atteignaient, en 1997, 5,4\% du P.N.B. - autant qu'aux Etats-Unis ou en Autriche (à Panama : 5,1\% au Nicaragua : 3,9\%, au Honduras : 3,6\% - source : P.N.U.D. Rapport mondial sur le développement humain 2000. Bruxelles : De Boeck Université, 2000. p. 194).

2. Jean-Marie AUBY, «La théorie du pouvoir de suffrage en droit constitutionnel français ", Politique, 1958, n4, p. 293 et s., spécialement p. 304.

3. Cf. Olivier DABENE, «Elections en Amérique centrale, 1989-1991 », Problèmes d'Amérique latine, 1991, 2, p. 3-20, spécialement p. 20.

4. Olivier DABENE, article précité, p. 19. 


\section{AUTEUR}

\section{CLAUDE EMERI}

Ancien professeur de Droit public

U.F.R. des Sciences Juridiques et Economiques de Guadeloupe

Université des Antilles et de la Guyane 